Mirjam Premrl Podobnik

Facoltà di Lettere e Filosofia

Università di Lubiana

Slovenia

mirjam.premrl@ff.uni-lj.si
UDK 811.131.1'36:811.163.6

DOI: $10.4312 /$ vestnik.13.127-146

\title{
GLI USI DELL'ARTICOLO E DEL PRO-AGGETTIVO IN ALCUNI TIPI DI SINTAGMI NOMINALI ITALIANI A CONFRONTO CON LO SLOVENO
}

INTRODUZIONE

Con il presente contributo desidero esporre alcuni dati legati all'uso cataforico e non dell'articolo e di alcuni pro-aggettivi ${ }^{1}$ in italiano. Mi interessa l'interdipendenza tra l'(in) determinatezza del nome testa (l'antecedente, il nucleo) e i complementi frasali o sintagmatici sottocategorizzati ${ }^{2}$ (i postmodificatori) che si suddividono in tre tipi: sintagmi sottocategorizzati (1), dipendenti relative (2), dipendenti completive (3).

(1) L'istituzione ${ }^{3}$ del portofranco non ha [...] effetti immediati sullo sviluppo della città: l'incremento dei traffici non raggiunge [...] (A/MI: 18)

(2) Ognuno si congedò dalla vita nel modo che più gli si addiceva. (L2I: 14)

(3) Ma nel lasciarla ho la netta sensazione che non ci andrò [...] (T2I: 10)

L'insieme del nome testa e dei suoi complementi è qui definito con il termine di sintagma nominale sottocategorizzante 4 (ad. es. sullo sviluppo della città).

Essere capaci di prevedere i possibili influssi dei postmodificatori sull'uso dell'articolo davanti al nucleo può essere utile facendo paragoni con lo sloveno, che come molte lingue slave dell'uso dell'articolo non dispone (cfr. Orožen 1972: 107; Vidovič-Muha 1996: 116, Bažec 2008: 235). Innanzitutto perché tale abilità potrebbe consentire a un parlante sloveno di italiano un'espressione grammaticalmente corretta, con ripercussioni positive sia nel campo di insegnamento/apprendimento che in quello di traduzione, ma

\footnotetext{
1 Il termine si riferisce agli elementi pronominali che nel testo coriferiscono con gli aggettivi (cfr. Beaugrande/ Dressler 1984: 77, 80-81), chiamati più comunemente aggettivi determinativi (possessivi, dimostrativi e indefiniti) (Dardano/Trifone 2011: 222-232).

2 Il termine è stato preso dall'opera di Renzi (2001: 419).

3 Le parti in corsivo evidenziano l'uso cataforico dell'articolo.

4 Il termine è stato coniato sul modello di sottocategorizzato per esprimere il concetto opposto.
} 
anche perché la capacità di osservare i testi nelle due lingue potrebbe permettere di fare luce sulle marche di cataforicità in sloveno, nonché sulla percezione della determinatez$\mathrm{za}$, in sloveno intesa come categoria extralinguistica. ${ }^{5}$

Nonostante l'assenza dell'articolo lo sloveno presenta alcuni mezzi morfolessicali (ad. es. i pro-aggettivi), che oltre ai significati particolari (Korzen 1996: 689-690, Vidovič-Muha 1996: 116) ne veicolano anche quello di (in)determinatezza. L'analisi qui esposta ha voluto scoprire anche il legame tra il tipo di pro-aggettivo (ta/tisti, takšen, isti, edini, njegov, kakšen) o il numerale ordinale e la (non)restrittività della frase relativa o completiva sottocategorizzata (vedi l'esempio ${ }^{6}(18)$ ). In italiano al posto del pro-aggettivo può comparire l'articolo (v. (26)).

La capacità di riconoscere la restrittività della dipendente in un testo sloveno aiuta a prevedere anche lo status di determinatezza dell'antecedente nel corrispondente testo italiano, e a riconoscere lo status di determinatezza dell'entità ${ }^{7}$ espressa con il sintagma sloveno. Ciò è più difficile quando l'antecedente nel testo sloveno non è preceduto da alcun pro-aggettivo (v. (34)). Immaginiamo di voler tradurre una struttura del genere dallo sloveno in italiano. In tale caso bisogna prendere in considerazione l'intreccio della (non) restrittività con altri fattori di co(n)testo e valutare il loro influsso sulla scelta dell'articolo, che alla fine può rivelarsi perfino non ascrivibile alla presenza del postmodificatore (v. (17)), quindi non un uso cataforico, o in casi di copresenza di più postmodificatori influenzata solo da uno di loro (v. (25)).

Un altro aspetto che si desidera sottolineare riguarda l'uso dell'articolo davanti ai postmodificatori sintagmatici: riconoscere il tipo di sintagma sottocategorizzante e quello sottocategorizzato aiuta a prevedere il tipo di articolo che verrà usato davanti al secondo (v. (5) e (7)).

L'analisi si è basata quindi sulle seguenti premesse:

a. La determinatezza è una categoria extralinguistica, quindi può essere riconosciuta e studiata anche in sloveno.

b. La capacità di riconoscere la determinatezza in sloveno aiuta ad usare meglio l'articolo in italiano.

c. Categorie e mezzi linguistici diversi dalla determinatezza, come la restrittività o la presenza dei complementi sottocategorizzati, possono aiutare a riconoscere, in base ai contenuti che veicolano e in base all'interpretazione del restante co(n)testo, lo status di determinatezza delle entità rese nel testo sloveno.

5 Oltre che con questa espressione la determinatezza può essere definita anche una categoria del senso, una categoria logico-pragmatica, una categoria del discorso e dei mondi testuali (Trenkić 2004: 1402; Beaugrande/Dressler 1984: 169; Lyons 1999: 1-15; Renzi 1976: 6; Schlamberger Brezar 2004: 35-36).

6 Da qui in poi al posto di vedi l'esempio sarà usata l'abbreviazione $v$.

7 Per quel che riguarda lo sloveno parlo di determinatezza del sintagma nominale soltanto in situazioni in cui sono presenti marche di determinatezza anche sul piano formale (ad. es. la presenza di pro-aggettivi). Quando invece essa non è marcata testualmente bensì inferita da altri elementi di co(n)testo (il contenuto, l'ordine dei costituenti, ecc.) mi riferisco allo status di determinatezza dell'entità verbalizzata con quel dato sintagma. 
Lo scopo specifico dell'analisi consiste nella presentazione e nel commento di alcuni passaggi dei testi scelti come corpus in cui la determinatezza interagisce con la catafora e la restrittività, e quello più generale nell'offrire agli sloveni dei mezzi che possano aiutarli ad usare meglio l'articolo in italiano.

L'articolo sarà strutturato nel seguente modo: dopo la presentazione del corpus e del metodo e la definizione della catafora saranno introdotti i complementi con (v. (7)) effetto cataforico e senza (v. (5)), seguiti dalla definizione della restrittività, dalla classificazione delle dipendenti in restrittive e non restrittive sulla base dell'elemento pronominale davanti al sintagma nucleo, e infine dall'analisi di esempi in cui si osserverà il legame tra l'(in) determinatezza e la (non)restrittività in combinazione con altri parametri di co(n)testo.

\section{2}

\section{CORPUS E METODO}

I risultati sono stati ottenuti con il metodo contrastivo e qualitativo esaminando l'espressione e la percezione della determinatezza e il suo legame con la catafora e la restrittività in un corpus di 14 testi letterari e non, contemporanei, di cui 10 originali italiani e 4 sloveni, e nelle rispettive traduzioni in sloveno e in italiano. Si sono esaminate le prime 20 pagine dell'originale e le rispettive pagine della traduzione. ${ }^{8}$ In entrambi i casi la varietà di lingua osservata è stata lo standard.

La scelta dei testi letterari e non letterari ${ }^{9}$ e la combinazione sempre diversa della coppia autore/traduttore ha consentito di cogliere una più vasta gamma d'uso linguistico, ma siccome lo scopo dell' analisi si limitava a riconoscere e a descrivere il legame tra la determinatezza e la catafora, e non a mettere a confronto i diversi tipi di testo o le coppie autore/traduttore per quel che riguarda i fenomeni studiati, nell'analisi queste distinzioni non sono state prese in considerazione. Di conseguenza non saranno marcate neppure nella presentazione dei risultati.

Secondo il metodo scelto l'autore è considerato un parlante ideale della lingua dell'originale, e il traduttore un parlante ideale sia della lingua di partenza che di quella di arrivo: conoscendo i mezzi linguistici del testo originale il traduttore interpreta il messaggio e si ricostruisce il relativo mondo testuale per renderlo successivamente nella lingua d'arrivo con i mezzi linguistici caratteristici dell'ultima (Coseriu 2002: 384-386). Basandosi su questa premessa l'analisi è partita sempre dal testo sloveno, indipendentemente dal fatto che fosse l'originale o la traduzione, cercando i sintagmi caratterizzati dal nucleo nominale seguito da un postmodificatore. Considerando la determinatezza una categoria

8 Uno degli esempi riportati è stato trovato più avanti nel libro, ma inserito nell'analisi perché rifletteva bene i fenomeni studiati.

9 L'elenco completo dei testi usati si trova alla fine dell'articolo nella sezione del Corpus. Le sigle indicanti ciascuna delle opere sono composte dall'iniziale del cognome dell'autore (seguita dal numero quando lo stesso autore compare due volte) e dall'indicazione della lingua del testo (I sta per italiano e S per sloveno). 
universale, anche se non verbalizzata con l'articolo, successivamente si è cercato di prevedere, in base al messaggio del sintagma sottocategorizzante e del co(n)testo, lo status di determinatezza delle entità rese con il nucleo, e nel caso dei sintagmi sottocategorizzati, anche lo status di determinatezza reso con essi. L'italiano con l'articolo rappresentava la lingua di controllo per le ipotesi fatte in base all'osservazione dei testi sloveni. Lo scopo di tale processo era duplice: predire l'uso dell'articolo in italiano e scoprire le spie di determinatezza in sloveno.

Con lo stesso metodo sono stati analizzati anche i casi di frasi relative in cui il nucleo è preceduto da un pro-aggettivo.

Tale metodo tuttavia presenta dei punti deboli: 1 . La scelta del corpus parallelo lascia spazio alla possibilità che il traduttore, influenzato dall'originale, con maggiore probabilità scelga lessico e strutture analoghi (ma sempre appropriati) a quelli dell'originale, allontanandosi così da quelli che sarebbero il lessico e le strutture di un testo autentico nella lingua d'arrivo (Ožbot 2012: 21). 2. La determinatezza è anche una categoria grammaticale (Lyons 1999: 276), il che significa che in lingue diverse è percepita e codificata in modo diverso. Con soli ragionamenti sulla determinatezza partendo dai testi sloveni perciò non si riesce sempre a prevedere l'uso dell'articolo in italiano.

\section{DEFINIZIONE DELLA CATAFORA}

Gli usi cataforici riguardano le situazioni in cui lo status di determinatezza del sintagma nucleo è influenzato dalla presenza di postmodificatori; sennonché lo sguardo sulla catafora sarebbe solo apparentemente esaustivo se ci si limitasse a definirla esclusivamente in base alla presenza dei postmodificatori nel testo di superficie. Sebbene secondo Korzen (1996: 72, 692) la catafora rientri tra gli usi dell'articolo motivati testualmente, Renzi (2001: 399) sottolinea i legami della catafora con il sapere extralinguistico dei partecipanti al discorso:

"Un caso particolare del tipo di determinatezza da conoscenze comuni è quello in cui l'identificazione del referente è ottenuta cataforicamente, cioè con precisazioni che seguono il $\mathrm{SN}$ in questione $[\ldots]$ :

(194) a. Sai che è stato chiuso il bar che è all'angolo di via Cavour?

b. Sai che l'inquilino di Carlo non paga l'affitto?

Nelle modificazioni dei SN che è all'angolo di via Cavour e di Carlo [...] possiamo vedere delle strategie per favorire l'identificazione di un referente già noto, ma che non potrebbe essere riconosciuto in modo inequivocabile senza delle precisazioni." 
La resa di determinati contenuti all'interno di un complemento sottocategorizzato sarebbe quindi una conseguenza delle intenzioni del parlante di assicurarsi il rinvio inequivocabile da parte del ricevente a un'entità a questi già nota e non in primo luogo la ragione in base a cui giudicare il sintagma nucleo determinato.

La presenza di un postmodificatore tuttavia non è a priori correlata con la presenza dell'articolo determinativo davanti al nucleo: gli enunciati degli esempi a. e b. funzionerebbero infatti anche con l'articolo indeterminativo (un bar/un inquilino) (Renzi ibid.). Nel presente contributo in tali casi si parlerà di pseudocatafora:

(4) [...] in 15 mesi di attività si meritò una promozione al grado di colonnello. (FI: 10)

[...] si je po petnajstih mesecih zaslužil [...] povišanje v polkovnika. (FS: 10)

Seguendo la teoria di Korzen (1996: 18), che vede nel sintagma introdotto dall'articolo zero l'espressione di un concetto, ${ }^{10} \mathrm{i}$ sintagmi senza l'articolo saranno detti complementi concettuali (d'orzo, pane, di granoturco). Questi non producono l'effetto cataforico, dal momento che formano un tutt'uno con il nucleo. L'intero sintagma è determinato se l'entità con esso resa è percepita come unica; può trattarsi di un'entità generalmente nota, oppure di un'entità individuante o parte di una massa (il caffè d'orzo) presentata come una sola in quel dato contesto (cfr. Korzen 1996: 690). ${ }^{11}$ In caso opposto l'entità non è determinata (un pezzo di pane di granoturco):

(5) Bevvero il caffè d'orzo, sgranocchiarono un pezzo di pane di granoturco [...] (ToI: 19-20)

Popili so ječmenovo kavo, poglodali kos koruznega kruha [...] (ToS: 6)

Ai livelli non avanzati di apprendimento di una lingua straniera il parlante non nativo (in)consciamente cerca di idearsi i contenuti prima nella propria lingua madre (o in

10 Il concetto/l'idea "racchiude /.../ delle proprietà tipiche secondo cui un insieme di oggetti può essere categorizzato" (Korzen 1996: 55). I concetti sono rappresentati da parole prive di co(n)testo (non attualizzate), ma la non-attualizzazione, marcata dall'articolo zero, può trovarsi anche all'interno di un testo: I nostri vicini di casa hanno tre figli. Con il sintagma sottocategorizzato di casa non si denota una casa concreta (un'entità), bensì un concetto (il tipo di vicini). Il sintagma di casa fa parte integrante del sintagma sovraordinato $i$ nostri vicini, ed unito ad esso, contribuisce a rendere completo il suo significato.

11 Un'entità (un concetto attualizzato, portatore di proprietà (Korzen 1996: 690)) è percepita come determinata se in un dato contesto è resa come unica o inclusa (un gruppo di entità che funziona come unico: $\boldsymbol{i}$ leoni dello zoo giacevano nell'ombra), familiare (nota agli interlocutori) o identificabile ("Passami il martello, per favore!": la frase rivolta a una persona appena entrata nella camera dall'altra già presente. La prima persona fino al momento della domanda non sapeva dell'esistenza del martello, ma ricevuto l'ordine sa di doversi guardare attorno per trovarlo.) (Lyons 1999: 1-15). 
un'altra lingua che già conosce), e solo successivamente li esprime in quella di arrivo (Ožbot 2009: 26). Essere in grado, come sloveni, di prevedere in base a un testo sloveno quali elementi saranno presentati sotto forma di complementi concettuali nel corrispondente testo italiano è utile perché così allo stesso tempo si risolve la questione della scelta dell' articolo. Il contenuto che in italiano sarà reso come complemento concettuale (d'orzo, di granoturco) in sloveno è a volte reso con un aggettivo (ječmenovo, koruznega). Se è realizzato con un nome, il sintagma sottocategorizzato è spesso al genitivo: kolonizacijski proces $=$ proces kolonizacije $\rightarrow$ processo di colonizzazione (LBS: 38/I: 39) (cfr. Toporišič 2004: 560). Ma come riportato da Bažec (2010/11: 49), i sintagmi sottocategorizzati sloveni (tra cui anche quelli concettuali) possono essere introdotti anche da preposizioni (iz ječmena) e seguiti da sintagmi declinati in casi diversi dal genitivo (s prtljažnikom). Anche in italiano il sintagma può essere introdotto da preposizioni diverse (a furgoncino) da quella più tipica di (di cacciatore):

(6) Bil je na triciklu s prtljažnikom. Ko je zagledal ptice, je začel močneje goniti [...] gnan od lovske slasti, čeprav v življenju ni imel v roki nobene druge puške, razen vojaške. (CS: 16)

Era su un triciclo a furgoncino, e vedendo gli uccelli pedalò più forte [...] preso da una fantasticheria di cacciatore, sebbene non avesse mai imbracciato altro fucile che quello del soldato. (CI: 18)

I contenuti del sintagma concettuale semanticamente delineano un (sotto)tipo di entità resa con il nucleo (un triciclo a furgoncino è un tipo di triciclo); sennonché anche quando in base alla realizzazione di un sintagma in sloveno (vojaška puška) ci aspetteremmo un sintagma concettuale in italiano, quello potrebbe non rivelarsi concettuale (fucile del soldato). Anche se la determinatezza viene percepita come una categoria extralinguistica del senso, in ogni lingua ha subito un processo di grammaticalizzazione in parte diverso (Lyons 1999: 276), per cui con soli ragionamenti sullo status di determinatezza di un'entità resa nel testo sloveno non si può sempre predire il tipo di articolo davanti al sintagma corrispondente nel testo italiano: gladovna stavka/sciopero della fame, avtobusno postajališčelfermata dell'autobus, terensko delo/lavoro sul campo (AS: 11, I: 5); poročni obred/cerimonia del matrimonio (AS: 15; I: 11), slovensko in nemško šolsko okoljelambiente delle scuole slovene e tedesche (A/MS: 20, I: 11).

Chiameremo argomentali (Salvi/Vanelli 2004: 153-154) i postmodificatori introdotti dall'articolo determinativo, ma anche dagli articoli indeterminativo o zero (v. (10)). ${ }^{12}$ Partendo dalla considerazione dei contenuti dei sintagmi nel testo sloveno, è possibile riconoscere alcuni elementi in base a cui tali sintagmi si possono ritenere argomenti e con

12 Oltre che di un concetto, l'assenza dell'articolo può essere segno anche di un'entità indeterminata al plurale (Korzen 1996: 195). 
ciò predire la presenza dell'articolo nel corrispettivo testo italiano. Le spie più evidenti di questo fenomeno sono soprattutto tre: a. con il nucleo è resa un'azione verbale sotto forma di nominalizzazione (7); b. con il nucleo è espresso l'agente (8); c. con il postmodificatore è espresso il possessore (9):

(7) Zaostritev političnih razmer [...] je spodbudila Mussolinija k nekaterim odločitvam. (FS: 16)

L'inasprimento della situazione politica [...] spinsero Mussolini ad adottare alcune contromisure. (FI: 16)

(8) Kristus [...] je hkrati darovano [...] in darovalec, ni pa izvajalec žrtvovanja. (AS: 21)

Cristo [...] è insieme l'offerta [...] e l'offerente, ma non l'esecutore del sacrificio. (AI: 18)

(9) Zgodovino koncentracijskih taborišč bomo narekovali mi. (L1S: 7)

La storia dei Lager, saremo noi a dettarla. (L1I: 3)

(10) Nobenega dvoma ni, da je bilo med začetkom tisočletja in 13. stoletjem obdobje velike rasti: o tem govorijo viri o obdelovanju novih površin [...] (LBS: 12)

Non è dubbia la fase di forte crescita tra l'inizio del millennio e il XIII secolo, testimoniata dalla messa a coltura di nuove terre [...] (LBI: 9) LA DETERMINATEZZA DEL SINTAGMA NUCLEO

Com'è generalmente noto le relative si distinguono in quelle restrittive e quelle non restrittive. Riportiamo la definizione di Cinque (2001: 458): "La frase relativa può avere due usi fondamentali. Essa può concorrere insieme all'antecedente, come parte integrante e insopprimibile, a individuare in modo univoco la persona o 1'oggetto [...] di cui si vuol parlare. Si tratta dell'uso ,restrittivo“ della frase relativa: per es. in Tutti quelli che ti hanno aiutato lo hanno fatto disinteressatamente. Oppure, la frase relativa può semplicemente aggiungere dell'informazione pertinente a proposito di una persona, o di un oggetto, già indipendentemente individuato: es. Tuo fratello, che mi è amico, mi ha avvertito subito."

Per Kordić (1995: 25) una restrittiva restringe i referenti rispetto a quelli che l'antecedente non modificato altrimenti denoterebbe. Cazinkić (2000/01: 31) condivide la posizione di Kordić, ritenendo che gli attributi restrittivi restringono il numero dei referenti del sintagma nominale, mentre quelli non restrittivi non esercitano alcun influsso su di essa. Le definizioni dei tre autori vorrebbero delineare gli stessi aspetti della restrittività, 
anche se le espressioni "individuare in modo univoco" e "restringere" non rappresentano lo stesso processo. Gli esempi delle restrittive citati da Cinque (2001: 459) riflettono più il concetto di "restringere":

(11) "Ho rivisto la ragazza che era con te ieri."

(12) "Ho rivisto una ragazza che avevo conosciuto dieci anni fa."

(13) "Cerco una ragazza che sappia cucinare.

(14) "Non c'erano purtroppo Ø amici che potessero aiutarmi."

Ma se come criterio della restrittività vera e propria si prende l'idea che l'antecedente debba formare un tutt'uno con la relativa (v. Cinque 2001: 458), ciò può essere confermato in (11), (13) e (14), dove l'articolo determina non soltanto l'antecedente, ma l'insieme dell' antecedente e della relativa. Non così in (12), dove l'articolo si riferisce più strettamente al nucleo, mentre le informazioni della relativa sono aggiunte per completare il messaggio. Dire quindi che la relativa restringe i referenti può essere valido anche in (12), ma l'articolo non restringe l'insieme del nucleo e della relativa. Di conseguenza in questo articolo saranno considerate propriamente restrittive soltanto quelle relative che sono parte integrante del nucleo. La struttura restrittiva può essere determinata come in (11), o indeterminata come in (13) e (14). Diremo, quindi, che tra quelle propriamente restrittive, come in (15), e quelle propriamente appositive, come in (16), che rappresentano i due prototipi, si ha un continuum di frasi intermedie, più o meno restrittive o appositive (cfr. Taylor 1995: xiv):

(15) Non so di quanto disprezzo sarei stato oggetto se avessi fatto la domanda che avevo sulla punta della lingua $[\ldots]$ (J2I: 6-7)

(16) Ho ripensato anche a Praga, dove una mattina dello scorso autunno [...] avevo vagato per la città deserta. (J1I: 9)

Una relativa non è restrittiva se sono presenti elementi di valutazione (lo ripeto):

(17) Le pagine di questo libro non indicano un rimedio di facile [...] attuazione. È già questa ammissione $[. .$.$] la dice lunga sulla natura del disagio che, lo ripeto,$ non è esistenziale ma culturale. (GI: 13)

\section{I PRO-AGGETTIVI COME DETERMINANTI E LA RESTRITTIVITÀ DELLA RELATIVA}

Data l'assenza dell' articolo, studiare il legame tra la catafora, la determinatezza e la restrittività in sloveno è difficile perché spesso non si raggiunge una conclusione convincente 
sullo status di determinatezza delle entità rese nel nucleo. Esaminare le situazioni in cui in sloveno il nucleo è preceduto da un pro-aggettivo, permette di fare luce su questo legame in modo esplicito e successivamente interpretare meglio lo status di determinatezza delle entità anche in assenza di pro-aggettivi. Per quel che riguarda lo sloveno, il fenomeno è stato discusso in Cazinkić (2000/01: 31-37). Le combinazioni che verranno esposte di seguito riflettono in parte le sue osservazioni, in parte, invece, si tratta degli esiti della presente analisi.

Quando davanti all'antecedente si trova il pro-aggettivo dimostrativo tisti/quello la relativa è restrittiva (cfr. ibid.: 35):

(18) Prva [...] pelje k analizi tistega sistema prisil, ki je vplival na demografske pojave $[\ldots]$ (LBS: 11)

Il primo [...] porta all' analisi di quel sistema di costrizioni che [...] ha condizionato i fenomeni demografici [...] (LBI: 8)

Ma se il dimostrativo fa parte di una ripresa anaforica la relativa è appositiva:

(19) Leta [...] nosim s seboj ta natančno opredeljeni občutek, ki ga varujem v sebi kot kakšno majhno odkritje [...] (T2S: 7)

Da anni cammino con questa precisa sensazione che covo dentro come una piccola scoperta [...] (T2I: 7-8)

(20) Nekakšni ptiči, rečne race [...] Eden tistih ptičev, za katere sem mislil, da so race, se je dvignil [...] (J1S: 9)

Degli insoliti uccelli, anatre di fiume [...] Uno degli uccelli che prima avevo pensato fossero anatre si è alzato [...] (J1I: 7-8).

È restrittiva la relativa che segue il nucleo introdotto dal pro-aggettivo dimostrativo isti/stesso:

(21) Seguirono la stessa via che avevano percorso per l'andata. (ToI: 25)

Šli sta po isti poti, kot sta prišli. (ToS: 12)

Allo stesso modo è restrittiva la relativa preceduta dal nucleo introdotto dal numerale ordinale o aggettivi quali unico, solo, ultimo, o il pro-aggettivo indefinito tutto (cfr. ibid.: 37):

(22) To je že tretja žena-opica, ki jo je odkril ta učenjak. (J1S: 13)

È la terza donna-scimmia scovata da questo scienziato. (J1I: 13) 
(23) Mislim, da sem edini človek, iz katerega se ni nikdar norčevala, edino bitje, ki mu je namenila popolnoma drugačno ravnanje. (T2S: 12)

La sola persona di cui non si sia mai presa gioco credo di essere io, l'unico essere che lei si sia proposta di trarre a sé totalmente. (T2I: 14)

(24) Izčrpen $[\ldots]$ prikaz bi moral $[. .$.$] zajeti celotno območje, za katero je [...]$ Ascoli skoval ime »Venezia Giulia« [...] (A/MS: 15-16)

Una storia completa $[\ldots]$ dovrebbe fare i conti $[\ldots]$ con tutta quella più vasta area per cui Ascoli $[\ldots]$ aveva coniato il termine di Venezia-Giulia $[\ldots]$ (A/ MI: 7)

In (25) il sintagma nucleo, introdotto da tutti/vsemi, subisce la restrizione e la determinatezza per mezzo del sintagma vstaje na Livadi, e non per mezzo della relativa, che è un'appositiva:

(25) Med vsemi zgodovinskimi voditelji vstaje na Livadi, ki so jih po njenem razpletu razselili po mnogih zaporih tedanje velike države [...] je gotovo užival največji ugled. (J2S: 6)

Tra tutti i capi storici della rivolta scoppiata nella Livada, a seguito della quale vennero spediti nelle numerose altre carceri dell'allora grande paese $[\ldots]$ era proprio lui a godere del maggior rispetto. (J2I: 6)

Anche se l'articolo/il pro-aggettivo indefinito un (un certo, neki) normalmente non determina l'insieme del nucleo e della frase relativa, nei casi in cui un è sinonimo di qualche/kakšen (cfr. ibid.: 37) succede l'opposto e la relativa è una restrittiva:

(26) [...] če je ona v kuhinji [...] moram tudi jaz najti kakšen opravek, ki bi vsaj deloma nadomestil delo v pisarni. (T2S: 12)

[...] se lei è di là che cucina [...] devo pure io trovare un'occupazione che sostituisca almeno in parte il lavoro d'ufficio. (T2I: 16)

La differenza tra i sintagmi introdotti da un sinonimo di qualche e un sinonimo di un certo sta nel fatto che nel primo caso si ha un'entità non-specifica (kakšen opravek), nel secondo invece un'entità specifica (o neki resnični, a nedoločljivi drugačnosti) (cfr. ibid.: 37):

(27) Tržaško identiteto [Slataper] prepoznava $v$ [...] sanjarjenju o neki resnični, a nedoločljivi drugačnosti, ki je pristna, ko se sramežljivo skriva v človekovi notranjosti, a postane zlagana, ko se sprevrže v proglašanje [...] (A/MS: 11) 
[...] Slataper [...] identifica la triestinità [...] col vagheggiamento di una diversità reale ma indefinibile, autentica quando viene vissuta nella pudica interiorità del sentimento e subito falsata quando viene proclamata [...] (A/ MI: 3)

In (27) la relativa, necessaria per il completamento della struttura e dei contenuti, si inserisce all'interno del continuum tra restrittive e appositive. In (28), invece, la relativa che segue il nucleo introdotto dal pro-aggettivo possessivo njegov è un'appositiva (cfr. ibid.: 36):

(28) Uradni Trst oziroma njegov vodilni razred, ki usmerja javno mnenje [...] odklanja Slataperja. (A/MS: 18)

La Trieste ufficiale ovvero la sua classe dirigente, che determina l'opinione della città, rifiuta [...] Slataper. (A/MI: 9)

Più difficili da classificare come restrittive o non sono le relative che seguono il nucleo introdotto dal pro-aggettivo indefinito tale/un/takšen, il cui significato è uguale a, ma non lo stesso (quindi indeterminato). Se come in (29) takšen compare in costrutti di ripresa anaforica, la relativa non è una restrittiva:

(29) Čisto po naključju se moški znajde sam z žensko v okoliščinah, ki ga [...] skoraj silijo k temu, da se loti avances spolnega značaja [...] Marsikateri moški misli, da mora izrabiti takšne 'priložnosti', ki so resnične ali domnevne [...] (T2S: 87-88).

Per puro caso un uomo si trova solo con una donna in circostanze tali che [...] quasi lo obbligano a compiere delle avances di natura sessuale [...] Molti uomini si credono in dovere di approfittare di simili 'occasioni', reali o presunte [...] (T2I: 126)

Ma in casi prototipici, come in (30), ci si accorge di trovarsi davanti a un costrutto ibrido, il cui significato potrebbe essere parafrasato nel seguente modo: con un'incredulità uguale a quell'incredulità che accuserebbe un lettore estraneo:

(30) V prvih dveh dneh sem jih trikrat [...] pregledal [...], danes pa še zmerom lahko trdim, da me navdajajo s prav takšno čudno nejevernostjo in prav takšno zadrego, kakršni bi prevzeli tujega bralca. (T2S: 12) ${ }^{13}$

13 L'originale italiano rende i contenuti determinati: $\grave{E}$ la terza [...] volta che li scorro [...] nel giro di questi due primi giorni, e oggi posso ancora affermare di ricavarne la stessa curiosa incredulità e lo stesso imbarazzo che vi accuserebbe un lettore estraneo. (T2I: 15-16) 
La parafrasi ci rivela così un costrutto che unisce il significato di indeterminatezza e di paragone a quello di ostensione e determinatezza. Il significato di indeterminatezza vorrebbe imporre una lettura non restrittiva della relativa: in questo senso la relativa non sarebbe modificata e determinata dal pro-aggettivo takšen assieme al nucleo. Dall'altro lato, vista la presenza del significato di ostensione, reso visibile nella parafrasi in cui la relativa funge da restrittiva, la relativa potrebbe essere interpretata come restrittiva.

\section{RESTRITTIVITÀ IN CORRELAZIONE CON LO STATUS DI DETERMINATEZZA IN ASSENZA DI PRO-AGGETTIVI DAVANTI AL NUCLEO}

Nella sezione 7 vengono presentati esempi con il sintagma nucleo, non preceduto da pro-aggettivi. Partendo dal testo sloveno si fanno delle ipotesi sullo status di determinatezza delle entità rese nel sintagma nucleo, in dipendenza da vari parametri tra cui la restrittività dell'elemento sottocategorizzato, la cataforicità, l'unicità e l'identificabilità dell'entità osservata, i contenuti espressi e il sapere extralinguistico. Si può scoprire, a volte, che la presenza dell'elemento sottocategorizzato non contribuisce alla scelta dell'articolo determinativo davanti al sintagma nucleo.

(31) Ona je sedla na foteljček ob vhodu [...] (T1S: 15)

Lei si era seduta sul divanetto dell'entrata [...] (T1I: 16)

Nell'interpretazione della determinatezza dell'entità foteljček entra in gioco il legame della catafora con il sapere extralinguistico, che qui non consiste nel fatto che il lettore destinatario conosca l'appartamento di cui si parla, bensì nella sua capacità di poterselo immaginare. Con il suo sapere generalizzato sulle più probabili dimensioni di un'anticamera e sulla quantità e la posizione di possibili divanetti si crea l'idea che sebbene nell'appartamento possano esistere più divanetti, quello dell'entrata sia probabilmente uno solo, e quindi lo considera determinato. L'originale italiano conferma l'ipotesi. Il sintagma $o b$ vhodu è restrittivo.

Anche in (32) il contenuto del sintagma pri sorodnikih attiva nel destinatario quel segmento del sapere extralinguistico secondo cui la camera presa in affitto presso paren$t i$ è in casi prototipici una sola:

(32) Čedalje redkeje sem prihajal $\mathbf{v}$ najemniško sobo pri sorodnikih [...] Večerja se je ponavadi zavlekla in čakala me je pripravljena postelja $\mathbf{v}$ sobi, $k j e r$ sem prespal neko noč, ko je bilo slabo vreme. (T2S: 16) 
Sempre più spesso dissertavo la camera d'affitto presso parenti [...] A cena si faceva tardi e io trovavo il letto pronto ad accogliermi nella stanza in cui ero rimasto in una notte di maltempo. (T2I: 21)

Allo stesso modo è una sola anche la stanza a casa della futura moglie, essendo connessa con un evento unico: il pernottamento in una notte di maltempo. La relativa è restrittiva e l'entità del sintagma nucleo determinata.

Ma la continuazione del sintagma nucleo con una relativa può non contribuire alla sua determinatezza. In (33) il suo contenuto ha una connotazione valutativa grazie all'elemento $k$ sreči/provvidenzialmente. Si considera l'entità hlače za dom determinata ragionando sul fatto che i pantaloni di casa rappresentano quell'unico paio di pantaloni (il protagonista altrimenti potrebbe averne di più) che, analogicamente allo slip, è stato indossato dal protagonista quella mattina, o il giorno prima. Il sintagma concettuale di casa crea un tutt'uno con il nucleo e con il suo contenuto precisa il tipo di pantalone fino a renderlo, presi in considerazione i ragionamenti esposti sopra, uno solo:

(33) Oblekel sem spodnjice, ki sem jih nosil že zjutraj, in hlače za dom, ki so $\boldsymbol{k}$ sreči visele v kopalnici. (T1S: 15)

Indossai lo slip smesso quella mattina, i pantaloni di casa pure provvidenzialmente appesi nel bagno. (T1I: 16)

Invece di contribuire alla determinatezza del nucleo, la presenza della relativa può persino permettere l'interpretazione indeterminata del nucleo, che in sua assenza sarebbe interpretato come determinato. Se in (34) si togliesse la relativa che segue il nucleo una borsetta, la borsetta sarebbe probabilmente determinata perché messa in relazione con la sua proprietaria. La relativa non è una restrittiva. Lo sarebbe se la donna avesse più borsette regalate con i soldi del primo articolo del protagonista e la borsetta in questione sarebbe una di esse. Solo in questo caso l'articolo una determinerebbe l'insieme del nucleo e della relativa. Ma in tal caso la struttura sarebbe diversa (una delle borsette che le regalai col mio primo articolo). Così, invece, la relativa soltanto aggiunge informazioni per descrivere la borsetta. Partendo dallo sloveno è difficile decidere se interpretare l' entità torbica come determinata o come indeterminata, dal momento che davanti al nucleo funzionerebbero sia neko che tisto (l'inserimento di uno o dell'altro serve a testare lo status di determinatezza). L'entità listek sarebbe invece percepita come indeterminata perché in termini della struttura informazionale risulta inaspettata e quindi inaccessibile (la donna potrebbe far cadere qualcos'altro) (cfr. Chafe 1994: 86). Anche qui la relativa aggiunge informazioni nuove a proposito del nucleo:

(34) [...] odpre torbico, ki sem ji jo podaril s honorarjem za svoj prvi objavljeni članek, in spusti na tla listek, na katerem je napisano: »Balkan.« (T2S: 12) 
[...] apre una borsetta che le regalai col mio primo articolo stampato e lascia cadere un biglietto sul quale è scritto "Balkan". (T2I: 12)

I sintagmi indeterminati compaiono spesso nelle funzioni predicative per dare informazioni nuove sulle entità del soggetto, oggetto o altri complementi (Korzen 1996: 104). Si potrebbe dire che servono a descrivere. In (35) la struttura ellittica nella variante italiana è un nome del predicato. La relativa non è una restrittiva, tuttavia è necessaria: grazie ai suoi contenuti la struttura ellittica acquista la necessaria salienza informazionale:

(35) Nato sem pomislil [...] da bosta odslej sami delno nosili naprej družinski priimek, ki bo izumrl z njima. Šlo je za star židovski rod, $k i$ se je razpotomčil zlasti v Grčiji. (T1S: 9)

Poi pensai che $[\ldots]$ portavano avanti a metà un nome di famiglia che si sarebbe estinto con loro. Un antico ceppo ebraico prosperato perlopiù in Grecia. (T1I: 9)

La capacità di descrivere non è caratteristica soltanto delle funzioni predicative. In (36) è descrittivo il sintagma in funzione di oggetto preposizionale. Come in (35) e (34), anche in (36) la lettura indeterminata del sintagma è possibile grazie alla relativa. Partendo dal testo sloveno è difficile decidere se considerare l'entità o družbi come determinata o no. Siccome di questa società si è parlato nel testo precedente l'interpretazione più naturale per uno sloveno sarebbe quella di considerare il nucleo determinato e la relativa una restrittiva, ma forse l'articolo indeterminativo nella variante italiana ci invita a considerare l'entità del nucleo indeterminata anche in sloveno:

(36) In kaj naj rečemo o družbi, ki ne izkoristi svoje biološke moči na višku [...] (GS: 10)

E che dire di una società che non impiega il massimo della sua forza biologica $[\ldots]$ (GI: 13)

Quando il sintagma nucleo è seguito da una completiva, essa lo completa in modo restrittivo, visto che il nucleo di per sé è incompleto (Prandi/De Santis 2011: 194). Il nucleo diventa determinato:

(37) Uveljavilo se je mišljenje, da je vsakdo, kdor ni kristjan [...] tujec in napadalec. (CaS:12).

Con esso, era radicata l'idea che chi non fosse cristiano [...] vi si fosse installato da estraneo e da invasore. (CaI: 8) 
In casi di copresenza di ripresa anaforica con il pronome dimostrativo e il complemento sottocategorizzato, la completiva è ugualmente una restrittiva:

(38) Tega še zmerom ne ve popolnoma [...] če dobro premislim, sem se spremenil prav zaradi te njene nesposobnosti, da bi vedela za takšne reči [...] (T2S: 9) Non lo sa interamente [...] a pensarci, è stata proprio questa sua incapacità di saperlo [...] ad avermi cambiato. (T2I: 11)

Non è però impossibile nemmeno una lettura indeterminata, difficile da predire se si parte dal testo sloveno:

(39) $[. .$.$] Slataper priznava [...] skušnjavo, da bi lagal. (A/MS: 11)$

Slataper confessa [...] una tentazione di mentire. (A/MI: 3)

La consapevolezza del fatto che in italiano il sintagma nominale che compare nel nome del predicato spesso è indeterminato ci può aiutare a predire, partendo dal testo sloveno, l'indeterminatezza di questo elemento, anche se seguito da una completiva:

(40) Ženska odvisnost pa je [...] pomirjujoča možnost, da se nanje lahko zanesemo $[\ldots]($ AS: 8)

La dipendenza delle donne invece è [...] una rassicurante possibilità di contare su di loro $[\ldots]$ (AI: 2$)$

\section{8}

\section{CONCLUSIONE}

Nell'articolo si è voluto indagare il legame tra la determinatezza del sintagma nucleo e i suoi complementi in italiano e in sloveno. La prima ad essere messa in relazione con la determinatezza è stata la catafora, che si ha quando i contenuti dei postmodificatori completano il significato del nucleo in modo tale da renderlo determinato. La seconda ad essere presentata è stata la restrittività del postmodificatore, che si ha quando il nucleo forma un tutt'uno con esso. In tal caso l'articolo o il pro-aggettivo, definiti o indefiniti, determinano l'insieme del nucleo e del postmodificatore, cioè tutto il sintagma sottocategorizzante. Se sono definiti e se il postmodificatore risulta restrittivo l'uso dell'articolo davanti al nucleo è cataforico. Per quel che riguarda le relative molte di loro si inseriscono in un continuum tra quelle restrittive e appositive. Tali dipendenti intermedie spesso apportano contenuti descrittivi che si combinano con l'indeterminatezza del nucleo. Le completive, che rendono il nucleo maggiormente determinato, possono comparire anche accanto a un nucleo indeterminato. 
I risultati sull'uso dell'articolo e dei pro-aggettivi in italiano confermano la possibilità di accedere alla comprensione della determinatezza attraverso lo sloveno. L'interpretazione dello status di determinatezza dell'entità resa nel nucleo con l'aiuto dei postmodificatori è possibile, ma, come previsto, per accedere al risultato positivo non è sufficiente affidarsi meccanicamente alla loro presenza nel testo: bisogna valutare i contenuti che essi arrecano in combinazione con gli elementi del rimanente co(n)testo. Lo stesso vale per la restrittività.

Tuttavia in alcuni casi (v. (34), (36), (39), o gli esempi della sezione 4) non si è avuta la certezza assoluta di poter ascrivere a un'entità nel testo sloveno lo stesso status di determinatezza che ha nel testo italiano, o, viceversa, di poter prevedere, partendo dal testo sloveno, l'uso dell'articolo in italiano.

L'analisi ha soltanto toccato alcuni aspetti del legame tra l'antecedente e il postmodificatore: lo status di determinatezza del complemento sintagmatico, il caso di antecedenti introdotti da pro-aggettivi e seguiti da dipendenti relative, e lo status di determinatezza dell'antecedente seguito da una relativa o da una completiva nelle situazioni in cui in italiano il sintagma sottocategorizzante è introdotto dall'articolo, mentre lo sloveno non presenta alcun elemento. Ciascuno di questi aspetti andrebbe approfondito (prendendo in considerazione anche il tipo testuale). Analisi ulteriori potrebbero riguardare anche il legame tra la determinatezza, la catafora e la funzione sintattica del sintagma sottocategorizzante, o l'interpretazione della determinatezza in casi di copresenza di più postmodificatori, comprese le situazioni in cui il nucleo nominale è preceduto o seguito da un aggettivo.

\section{CORPUS}

AI: ACCATI, Luisa (1998) Il mostro e la bella. Padre e madre nell'educazione cattolica dei sentimenti. Milano: Cortina Raffaello.

AS: ACCATI, Luisa (2001) Pošast in lepotica: oče in mati v katoliški vzgoji čustev. Trad. I. Prosenc. Ljubljana: Studia humanitatis.

A/MI: ARA, Angelo/Claudio MAGRIS (2007) Trieste. Un'identità di frontiera. Torino: Einaudi.

A/MS: ARA, Angelo/Claudio MAGRIS (2001) Trst. Obmejna identiteta. Trad. M. Cenda Klinc. Ljubljana: Študentska založba.

CI: CALVINO, Italo (1963) Marcovaldo ovvero le stagioni in città. Torino: Einaudi.

CS: CALVINO, Italo (1987) Marcovaldo ali letni časi v mestu. Trad. E. Umek. Ljubljana: MK.

CaI: CARDINI, Franco (2001) Europa e islam: storia di un malinteso. Roma/Bari: Laterza. 
CaS: CARDINI, Franco (2003) Evropa in islam: zgodovina nekega nesporazuma. Trad. M. Bajt. Ljubljana: Založba *Cf.

FS: $\quad$ FERENC, Tone (1999) »Ubija se premalo«. Obsojeni na smrt-talci-ustreljeni v Ljubljanski pokrajini 1941-1943. Dokumenti. Ljubljana: INZ: Društvo piscev zgodovine NOB.

FI: $\quad$ FERENC, Tone (1999)»Si ammazza troppo poco«. Condannati a morte-ostaggi - passati per le armi nella provincia di Lubiana 1941-1943. Documenti. Trad. P. Kodrič. Ljubljana: Istituto per la storia moderna: Società degli scrittori della storia della Lotta di Liberazione.

GI: GALIMBERTI, Umberto (2007) L'ospite inquietante: il nichilismo e i giovani. Milano: Feltrinelli.

GS: GALIMBERTI, Umberto (2009) Grozljivi gost: nihilizem in mladi. Trad. V. Simoniti. Ljubljana: Modrijan.

J1S: JANČAR, Drago (1984) Severni sij. Murska sobota: Pomurska založba.

J1I: JANČAR, Drago (2008) Aurora boreale. Trad. D. Betocchi/E. Lenaz. Milano: Bompiani.

J2S: JANČAR, Drago (1998) Zvenenje v glavi. Ljubljana: MK.

J2I: JANČAR, Drago (2007) Il ronzio. Trad. R. Dapit/M. Vidali. Udine: Forum.

L2I: LEVI, Primo (1996) Se questo è un uomo. La tregua. Torino: Einaudi.

L2S: LEVI, Primo (1996) Ali je to človek. Premirje. Trad. S. Šlenc. Ljubljana: Cankarjeva založba.

L1I: LEVI, Primo (1990) I sommersi e i salvati. Torino: Einaudi.

L1S: LEVI, Primo (2003) Potopljeni in rešeni. Trad. I. Prosenc Šegula. Ljubljana: Studia Humanitatis.

LBI: LIVI BACCI, Massimo (1998) La popolazione nella storia d'Europa. Roma/Bari: Laterza.

LBS: LIVI BACCI, Massimo (2007) Prebivalstvo v zgodovini Evrope. Trad. V. Troha. Ljubljana: Založba *Cf.

T2I: TOMIZZA, Fulvio (1972) La città di Miriam. Milano: Mondadori.

T2S: TOMIZZA, Fulvio (1974) Miriamino mesto. Trad. S. Ivanc. Ljubljana: DZS.

T1I: TOMIZZA, Fulvio (2000) La visitatrice. Milano: Mondadori.

T1S: TOMIZZA, Fulvio (2005) Obiskovalka. Trad. M. Košuta. Celovec: Mohorjeva družba.

ToS: TOMŠIČ, Marjan (1991) Šavrinke. Ljubljana: ČZP Kmečki glas.

ToI: TOMŠIČ, Marjan (1991) Le saurine. Trad. D. Fabjan Bajc. Koper: Zgodovinsko društvo za južno Primorsko: Znanstveno raziskovalno središče RS. 


\section{BIBLIOGRAFIA}

BAŽEC, Helena (2008) Sull'articolo determinativo sloveno. Linguistica e Filologia 26, 235-256).

BAŽEC, Helena (2010/11) La nascita degli articoli nello sloveno: settore scientifico-disciplinare di afferenza: scienze del linguaggio (linguistica teorica generativa): tesi di dottorato. Benetke: Helena Bažec. 30. junij 2014. http://dspace.unive.it/bitstream/ handle/10579/1124/Dottorato_Helena_Bazec.pdf?sequence=1.

CAZINKIĆ, Robert (2000/01) Oziralni prilastkovi odvisniki. Jezik in slovstvo 46/1-2, $29-40$.

CHAFE, Wallace L. (1994) Discourse, consciousness and time. The Flow and Displacement of Conscious Experience in Speaking and Writing. Chicago/London: The University of Chicago Press.

CINQUE, Guglielmo (2001) La frase relativa. L. Renzi e al. (cur.), Grande grammatica italiana di consultazione. Vol. 1. Bologna: il Mulino, 457-517.

COSERIU, Eugenio (2002) Napačna in pravilna izhodišča v teoriji prevajanja. Trad. M. Ožbot. M. Ožbot (cur.), Prevajanje srednjeveških in renesančnih besedil: 27. prevajalski zbornik. Ljubljana: Društvo slovenskih književnih prevajalcev, 380-397.

DARDANO, Maurizio/Pietro TRIFONE (2011) Grammatica italiana: con nozioni di linguistica. Bologna: Zanichelli.

DE BEAUGRANDE, Robert-Alain/Wolfgang Ulrich DRESSLER (1984) Introduzione alla linguistica testuale. Trad. Silvano Muscas. Bologna: il Mulino.

KORDIĆ, Snježana (1995) Relativna rečenica. Zagreb: Hrvatsko filološko društvo. Matica hrvatska.

KORZEN, Iørn (1996) L'articolo italiano fra concetto ed entità: uno studio semantico-sintattico sugli articoli e sui sintagmi nominali italiani con e senza determinante - con un'indagine particolare sulla distribuzione del cosiddetto »articolo partitivo". København: Museum Tusculanum Press.

LYONS, Christopher (1999) Definiteness. Cambridge: Cambridge University Press.

PRANDI, Michele/Cristiana DE SANTIS (2015) Le regole e le scelte: manuale di linguistica e di grammatica italiana. Torino: UTET Università.

OROŽEN, Martina (1972) K določnemu členu v slovenščini. Slavistična revija 20/1-4, $105-114$.

OŽBOT, Martina (2009) Nekaj kontrastivnih beležk o italijanščini in slovenščini in nekaj opažanj o jezikovni produkciji pri govorcih slovenščine v Italiji. Jezik in slovstvo $54 / 1,25-47$.

OŽBOT, Martina (2012) Prevodne zgodbe: poskusi z zgodovino in teorijo prevajanja s posebnim ozirom na slovensko-italijanske odnose. Ljubljana: Založba ZRC, ZRC SAZU. RENZI, Lorenzo (1976) Grammatica e storia dell'articolo italiano. Studi di grammatica italiana $\mathrm{V}, 5-42$. 
RENZI, Lorenzo (2001) L'articolo. L. Renzi et al. (cur.), Grande grammatica italiana di consultazione. Vol. 1. Bologna: il Mulino, 371-437.

SALVI, Giampaolo/Laura VANELLI (2004) La nuova grammatica italiana. Bologna: il Mulino.

SCHLAMBERGER BREZAR, Mojca (2004) Diskurzivni pristop k problemu določnosti V slovenščini. Jezik in slovstvo 49/5, 35-44.

TAYLOR, John R. (1995) Linguistic Categorization. Prototypes in Linguistic Theory. Oxford: Clarendon Press.

TOPORIŠIČ, Jože (2004) Slovenska slovnica. Maribor: Obzorja.

TRENKIĆ (2004) Definiteness in Serbian/Croatian/Bosnian and some implications for the general structure of the minimal phrase. Lingua 114, 1401-1427.

VIDOVIČ-MUHA, Ada (1996) Določnost kot besedilna prvina v slovničnem opisu slovenskega jezika (ob Kopitarjevi slovnici). J. Toporišič (cur.), Jernej Kopitar in njegova doba-Obdobja 15. Kopitarjev zbornik: mednarodni simpozij v Ljubljani, 29. junij do 1. julij 1994: simpozij ob stopetdesetletnici njegove smrti. Ljubljana: Filozofska fakulteta, Oddelek za slovanske jezike in književnosti, Seminar slovenskega jezika, literature in kulture, 115-130.

\section{POVZETEK}

\section{RABA ČLENA IN ZA-PRIDEVNIKA V NEKATERIH VRSTAH ITALIJANSKIH SAMO- STALNIŠKIH SINTAGEM V PRIMERJAVI S SLOVENŠČINO}

Članek se osredotoča na razmerje med podrednim dopolnilom, t. j. samostalniško sintagmo, relativnim ali vsebinskim odvisnikom, in rabo člena ali za-pridevnika v nadredni, jedrni samostalniški sintagmi. Predstavljeni so izsledki kvalitativne analize korpusa slovenskih in italijanskih besedil ter njihovih prevodov v italijanščino oz. slovenščino, katere namen je bil razpoznavati indice za določnost entitet $\mathrm{v}$ slovenščini in predvideti rabo člena $\mathrm{v}$ italijanščini ter tako pokazati možnosti, ki jih ima Slovenec za ustrezno izražanje v italijanščini. Ob predpostavkah, da je določnost univerzalna kategorija in zato razpoznavna tudi v jezikih, ki je ne zaznamujejo s členom (slovenščina), ter da sta avtor in prevajalec idealna govorca slovenščine in/ali italijanščine, je kot izhodišče vsakokrat služilo slovensko besedilo, italijansko pa je imelo kontrolno vlogo. Raba člena je opredeljena kot kataforična, če vsebina dopolnila pripomore k določni interpretaciji jedra. Podredne samostalniške sintagme se delijo na konceptualne in argumentalne. V italijanščini so prve zaznamovane $\mathrm{z}$ ničtim členom, z njimi pa je izražena ne-entiteta, z jedrom tvorijo pomensko enoto; druge pa, ki so zaznamovane s katerimkoli členom (tudi ničtim) in so izraz entitete, pomenske enote ne tvorijo. $\mathrm{Z}$ določnostjo je povezana restriktivnost odvisnika (relativnega ali vsebinskega), ki je v tem, da člen ali za-pridevnik določata celoto jedra in podrednega dopolnila. Jedro je lahko določno ali nedoločno. Pri nerestriktivnosti je odvisnik (relativni) le neobvezni dodatek. Analiza je pokazala, da se 
nekateri relativni odvisniki umeščajo med oba pola. Ti odvisniki pogosto prinašajo opisno vsebino, njihova pa odnosnica je nedoločna. Komentirani so zgledi, kjer je v slovenščini odnosnica uvedena z za-pridevnikom, in zgledi, kjer pred odnosnico ni določevalnika.

Ključne besede: člen, za-pridevnik, določnost, katafora, restriktivnost

\section{ABSTRACT \\ THE USES OF ARTICLES AND PRO-ADJECTIVES IN CERTAIN TYPES OF ITALIAN NOUN PHRASES IN COMPARISON WITH SLOVENE}

The article focuses on the relationship between postmodifiers in the form of noun phrases, relative and content clauses, and the use of articles or pro-adjectives in the nominal heads. The results of a qualitative analysis of Slovene and Italian texts and their translations into Italian and Slovene are presented, the main purpose of which was to identify markers of definiteness in Slovene and to predict the use of articles in Italian, thus showing the possibilities for Slovenes to express themselves appropriately in Italian. Assuming that definiteness is a universal category and therefore recognisable also in languages without articles (Slovene), and considering the author and the translator ideal speakers of Slovene and/or Italian, the Slovene texts served as the starting point of each analysis, while the Italian texts played the role of control. An article use is defined as cataphoric if the content of the postmodifier contributes to the definite interpretation of its head. Subordinate noun phrases can be divided into conceptual and argumentative. In Italian, the former, expressing a non-entity, are marked by a zero article and form a semantic unit with their heads, whereas the latter, expressing an entity, are marked by an article (included the zero one) and do not form a semantic unit with their heads. Related to definiteness is the restrictiveness of the clause, which consists in the article or pro-adjective determining the head including its postmodifier. Such heads can be both definite or indefinite. The analyses have shown instances of relative clauses that are placed between restrictive and non-restrictive ones. Conveying descriptive information, they occur after the heads preceded by an indefinite article. The definiteness of nominal heads preceded by a pro-adjective or without a determiner in Slovene texts is also discussed.

Keywords: articles, pro-adjectives, definiteness, cataphora, restrictiveness 